PHYSICAL REVIEW A 80, 022114 (2009)

\title{
State reconstruction by on/off measurements
}

\author{
Alessia Allevi \\ CNISM UdR Milano, I-20133 Milano, Italy \\ Alessandra Andreoni \\ Dipartimento di Fisica e Matematica, Università degli Studi dell'Insubria, I-22100 Como, Italy \\ and CNISM UdR Como, I-22100 Como, Italy \\ Maria Bondani* \\ ULTRAS, CNR-INFM, I-22100 Como, Italy \\ Giorgio Brida, Marco Genovese, ${ }^{\dagger}$ Marco Gramegna, and Paolo Traina \\ Istituto Nazionale di Ricerca Metrologica, I-10135 Torino, Italy \\ Stefano Olivares \\ CNISM UdR Milano, I-20133 Milano, Italy and Dipartimento di Fisica, Università degli Studi di Milano, I-20133 Milano, Italy \\ Matteo G. A. Paris \\ Dipartimento di Fisica, Università degli Studi di Milano, I-20133 Milano, Italy; \\ CNISM UdR Milano, I-20133 Milano, Italy; \\ and ISI Foundation, I-10133 Torino, Italy \\ Guido Zambra \\ Dipartimento di Elettronica, Politecnico di Milano, I-20133 Milano, Italy
}

(Received 27 February 2009; published 18 August 2009)

\begin{abstract}
We demonstrate a state reconstruction technique which provides either the Wigner function or the density matrix of a field mode and requires only avalanche photodetectors, without any phase or amplitude discrimination power. It represents an alternative to quantum homodyne tomography of simpler implementation.
\end{abstract}

DOI: $10.1103 /$ PhysRevA.80.022114

PACS number(s): 03.65.Wj, 42.50.Ar, 42.50.Dv

\section{INTRODUCTION}

The characterization of states and operations at the quantum level plays a leading role in the development of quantum technology. A state reconstruction technique is a method that provides the complete description of a physical system upon the measurements of an observable or a set of observables [1]. An effective reconstruction technique gives the maximum possible knowledge of the state, thus allowing one to make the best, at least the best probabilistic, predictions on the results of any measurement that may be performed on the system. At a first sight, there is an unavoidable trade-off between the complexity of the detection scheme and the amount of extractable information, which can be used to reconstruct the quantum state [2]. Currently, the most effective quantum state reconstruction technique for the radiation field is quantum homodyne tomography (QHT) [3,4], which requires the measurement of a continuous set of field quadrature and allows for the reliable reconstruction of any quantity expressible in terms of an expectation value [5-10]. A question arises on whether the trade-off may be overcome by a

\footnotetext{
*maria.bondani@uninsubria.it

†m.genovese@inrim.it

†matteo.paris@ fisica.unimi.it
}

suitable experimental configuration or it corresponds to some fundamental limitations. Here we demonstrate that no specific discrimination power is required to the detector in either amplitude or phase and that full state reconstruction is possible by a suitable processing of the data obtained with detectors revealing light in the simplest way, i.e., on/off detectors, such as single-photon avalanche photodiodes. Of course, some form of phase and/or amplitude modulation is necessary, which, in our scheme, is imposed to the field before the detection stage. In fact, our technique is built on the completeness of any set of displaced number states [11-16] and the reliable maximum likelihood reconstruction of arbitrary photon-number distributions [17] from on/off data.

The paper is structured as follows. In Sec. II we describe our reconstruction method, whereas in Sec. III the experimental setup used in the reconstruction is described in some details. Results are illustrated in Sec. IV and the error analysis is reported in Sec. V. In Sec. VI we discuss few additional topics while Sec. VII closes the paper with some concluding remarks.

\section{STATE RECONSTRUCTION BY ON/OFF MEASUREMENTS}

We start to describe our reconstruction technique by observing that the modulation of a given signal, described by 
the density matrix $\varrho$, corresponds to the application of a coherent displacement (probe) $\varrho_{\alpha}=D(\alpha) \varrho D^{\dagger}(\alpha), \alpha \in \mathrm{C}$. In practice, it can be easily obtained by mixing the state under investigation with a known coherent reference in a beam splitter (BS) or a Mach-Zehnder interferometer [18]. Upon varying amplitude and phase of the coherent reference and/or the overall transmissivity of the interferometer, the modulation may be tuned in a relatively broad range of values. The main idea behind our method is simple: the photon distributions of coherently modulated signals, i.e., the diagonal elements $p_{n}(\alpha)=\left\langle n\left|\varrho_{\alpha}\right| n\right\rangle$ of the density matrix $\varrho_{\alpha}$, contain relevant information about the complete density matrix of the original signal $\varrho$. Upon measuring or reconstructing the photon distribution $p_{n}(\alpha)$ for different values of the modulation one has enough information for full state reconstruction. By rewriting the above relation as $p_{n}(\alpha)$ $=\sum_{k m} D_{n k}(\alpha) \varrho_{k m} D_{m n}(\alpha)$, the off-diagonal matrix elements may be recovered upon inversion by least-squares method, i.e., $[14]$

$$
\langle m+s|\varrho| m\rangle=N_{\phi}^{-1} \sum_{l=1}^{N_{\phi}} \sum_{n=0}^{\bar{n}} F_{n m}^{(s)}(|\alpha|) p_{n}\left(|\alpha| e^{i \phi_{l}}\right) e^{i s \phi_{l}},
$$

where $N_{\phi}$ is the number of modulating phases, $\bar{n}$ is the truncation dimension of the Fock space, and $F$ depends only on $|\alpha|$ [14]. State reconstruction by the above formula requires, in principle, only phase modulation of the signal under investigation. Maximum likelihood methods and iterative procedures may be also used [19]. On the other hand, the Wigner function may be reconstructed using its very definition in terms of displacement [20],

$$
W(\alpha)=\operatorname{Tr}\left[D(\alpha) \varrho D^{\dagger}(\alpha)(-1)^{a^{\dagger} a}\right]=\sum_{n}(-1)^{n} p_{n}(\alpha) .
$$

As a matter of fact, the measurement of the photon distribution is challenging as photodetectors that can operate as photon counters are rather rare and affected either by a low quantum efficiency [21] or require cryogenic conditions, thus impairing common use [22,23]. Therefore, a method with displacement but without photocounting has been used so far only for states in the $0-1$ subspace of the Fock space [16]. On the other hand, the experimental reconstructions of photon-number distributions for both continuous-wave and pulsed light beams are possible using simple on/off singlephoton avalanche photodetectors. This requires the collection of the frequencies of the off events, $P_{0, k}=\sum_{n=0}^{\infty}\left(1-\eta_{k}\right)^{n} p_{n}$, at different quantum efficiencies of the detector, $\eta_{k}$. The data are then used in a recursive maximum likelihood reconstruction algorithm that yields the photon-number distributions as

$$
p_{n}^{i+1}=p_{n}^{i} \sum_{k=1}^{K}\left(A_{k n} / \sum_{j} A_{k j}\right)\left(P_{0, k} / p_{0, k}\left[\left\{p_{n}^{i}\right\}\right]\right),
$$

where $A_{k n}=\left(1-\eta_{k}\right)^{n}$ and $p_{0, k}$ is the probability of off events calculated from the reconstructed distribution at the $i$ th iteration [24]. The effectiveness of the method has been demonstrated for single-mode [17] and multimode fields [25] and also applied to improve quantum key distribution [26].

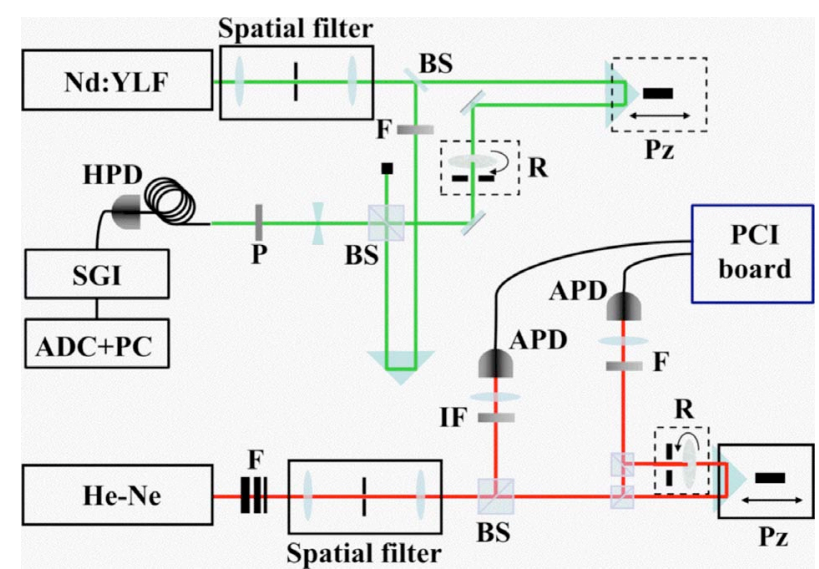

FIG. 1. (Color online) Schematic diagram of the two experimental setups. Upper panel: Nd:YLF, pulsed laser source; P, polarizer; HPD, hybrid photodetector; SGI, synchronous gated integrator; and ADC, analog-to-digital converter. Lower panel: He-Ne, cw laser source; IF, interference filter; and APD, single-photon avalanche photodiode. F, neutral-density filter; BS, beam splitter; Pz, piezoelectric movement; and R, rotating ground glass plate. Components in dotted boxes are inserted or activated when necessary.

Since the implementation of the modulation is relatively easy, we have thus a reconstruction technique which provides the quantum state of radiation modes and requires only avalanche detectors, without any phase or amplitude discrimination power. Here, we develop the idea into a proper reconstruction technique and demonstrate the reconstruction of the Wigner function [27] and the density matrix for different states of the optical field.

\section{EXPERIMENTAL SETUPS}

We have performed two experiments for the reconstruction of the Wigner function and the density matrix. In Fig. 1 we sketch the corresponding experimental setups: the upper panel for the measurement of the Wigner function and the lower panel for the density matrix. The first set of measurements was performed on ps-pulsed light fields at $523 \mathrm{~nm}$ wavelength. The light source was the second-harmonic output of a Nd:YLF mode-locked laser amplified at $5 \mathrm{kHz}$ (High $Q$ Laser Production) delivering pulses of $\sim 5.4$ ps duration. The field, spatially filtered by means of a confocal telescope, was split into two parts to give both signal and probe. The photon-number distribution of the probe was kept Poissonian, while the coherent photon-number distribution of the signal field was modified in order to get suitable states of light. In particular, we have generated two phase-insensitive classical states, namely, a phase-averaged coherent state and a single-mode thermal state. The first one was obtained by changing the relative phase between signal and probe fields at a frequency of $\sim 300 \mathrm{~Hz}$ by means of a piezoelectric movement ( $\mathrm{Pz}$ in the upper panel of Fig. 1), covering $1.28 \mu \mathrm{m}$ span. On the other hand, to get the single-mode thermal state we inserted Arecchi's rotating ground glass disk on the pathway of the signal field followed by a pin hole that selected a single coherence area. 
Signal and probe fields were mixed in an unpolarizing cube BS and a portion of the exiting field was sent, through a multimode optical fiber (600 $\mu \mathrm{m}$ core diameter), to a hybrid photodetector module (HPD mod. H8236-40, Hamamatsu, maximum quantum efficiency $\eta_{\mathrm{HPD}}=0.4$ at 550 $\mathrm{nm})$. Although the detector is endowed with partial photon resolving capability, we used it as a simple on/off detector by setting a threshold at the value corresponding to zero detected photons. Its output current pulses were suitably gate integrated by a SR250 module (Stanford Research Systems, $\mathrm{CA}$ ) and sampled to produce a voltage, which was digitized and recorded at each shot. In order to modulate the amplitude of the probe field, a variable neutral-density filter was placed on its pathway. The maximum overall detection efficiency, calculated by including the losses of the collection optics, was $\eta_{\max }=0.29$. We used a polarizer put in front of the fiber to vary the quantum efficiency of the detection chain from $\eta_{\max }$ to 0 .

\section{RESULTS}

Here we illustrate the reconstruction obtained for the Wigner function and the density matrix of phase-averaged coherent states and thermal states, as obtained by our method after recording the on/off statistics of amplitude- and/or phase-modulated signals.

In Fig. 2 we report the reconstructed Wigner functions for a phase-averaged coherent state with real amplitude $z \simeq 2.1$ and a thermal state with average number of photons $n_{t h}$ $\simeq 2.4$. The Wigner function of the vacuum state is also reported for comparison. As it is apparent from the plots all the relevant features of the Wigner functions are well recovered, including oscillations and the broadening due to thermal noise. In this case raw data are frequencies of the off event (collected over 30000 laser shots) as a function of detector efficiency, taken at different amplitudes of the modulating field, whereas the intermediate step corresponds to the reconstruction of the $p_{n}(\alpha)$. The insets of Fig. 2 display $p_{n}(\alpha)$ for the phase-averaged coherent state at two values of the modulation.

The second set of measurements has been performed to achieve state reconstruction with phase modulation. Here the light source was a He-Ne laser beam attenuated to singlephoton regime by neutral-density filters. The spatial profile of the beam was purified from non-Gaussian components by a spatial filter. Beyond a beam splitter, part of the beam was addressed to a control detector in order to monitor the laser amplitude fluctuations, while the remaining part was sent to a Mach-Zehnder interferometer. A piezomovement system allowed changing the phase between the "short" and "long" paths by driving the position of the reflecting prism with nanometric resolution and high stability. The beam on the short arm represented the probe, while the beam on the long arm was the state to be reconstructed. In the first acquisition the signal was the coherent state itself, while in the second acquisition it was a pseudothermal state generated as described above. The detector, a Perkin-Elmer single-photon avalanche photodiode (SPCM mod. AQR-12, Perkin Elmer) with quantum efficiency $\eta_{\max }=0.67$, was gated by a $20 \mathrm{~ns}$
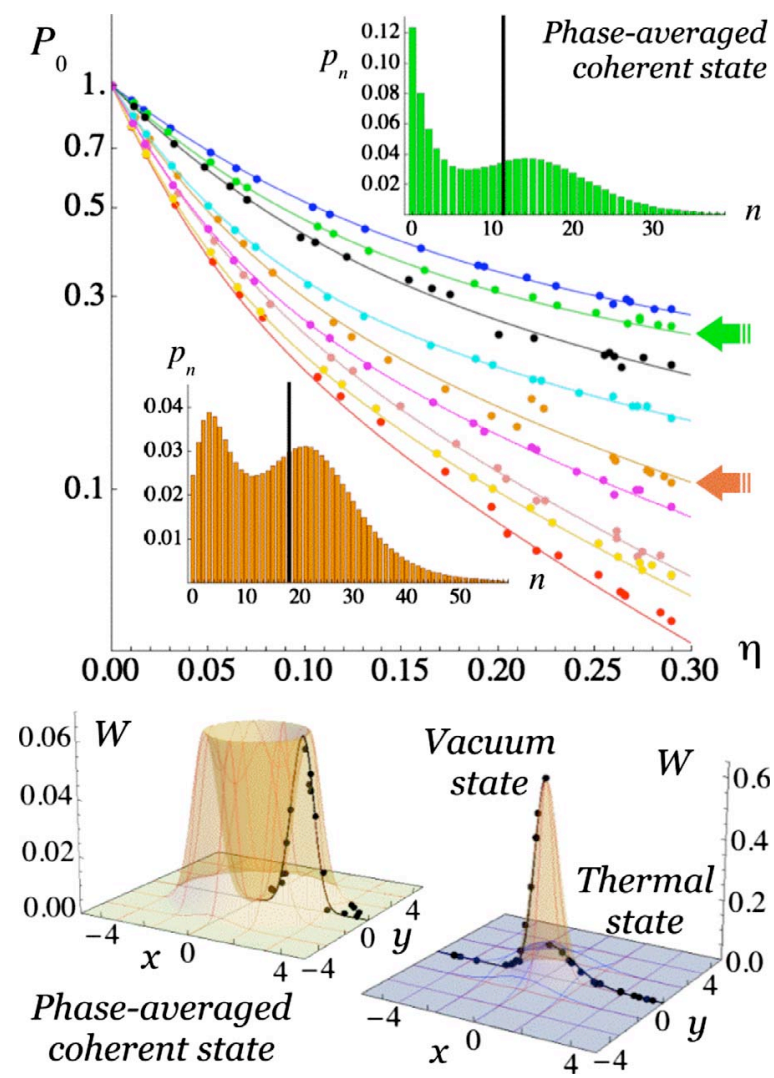

FIG. 2. (Color online) State reconstruction by amplitudemodulation and on/off measurements. In the main plot we report the off frequencies as a function of the quantum efficiency as obtained when the signal under investigation is a phase-averaged coherent state and for different values of the probe intensity $|\alpha|^{2}$. The two insets show the reconstructed photon distributions for $|\alpha|^{2}=5.02$ and $|\alpha|^{2}=10.69$, corresponding to the off distributions indicated by the arrows. The vertical black bars denote the mean value of the photon number for the two distributions $\left(\left\langle a^{\dagger} a\right\rangle=11.3\right.$, upper and $\left\langle a^{\dagger} a\right\rangle=18.0$, lower). In the lower left plot we report the corresponding reconstructed Wigner function. In the lower right plot we report the Wigner functions for signals in (blue) thermal state and (yellow, with sharper peak) vacuum.

time window at a repetition rate of $200 \mathrm{kHz}$. To obtain a reasonable statistics, a single run consisted of five repetitions of acquisitions lasting $4 \mathrm{~s}$ each. In the bottom part of Fig. 3 we report the reconstructed density matrix in the Fock representation (diagonal and subdiagonal) for a coherent state with real amplitude $z \simeq 1.8$ and a thermal state with average number of photons equal to $n_{t h} \simeq 1.4$.

As it is apparent from the plots the off-diagonal elements are correctly reproduced in both cases despite the limited visibility. Here the raw data are frequencies of the off event as a function of the detector efficiency, taken at different phase modulations, $\phi$, whereas the intermediate step corresponds to the reconstruction of the photon distribution for the phase-modulated signals. In our experiments we used $N_{\phi}$ $=12$ and $|\alpha|^{2}=0.01$ for the coherent state and $|\alpha|^{2}=1.77$ for the thermal state. The use of a larger $N_{\phi}$ would allow the reliable reconstruction of far off-diagonal elements, which is not possible in the present configuration. In the insets of Fig. 


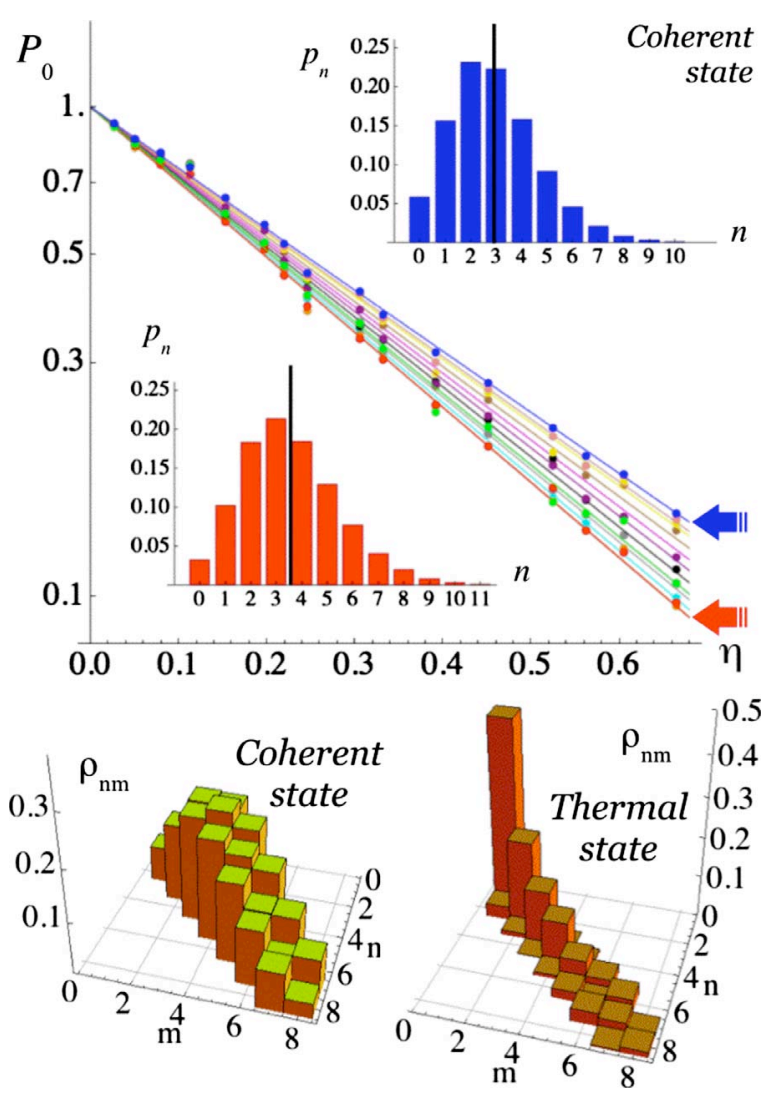

FIG. 3. (Color online) State reconstruction by phase-modulation and on/off measurements. In the upper plot we report the off frequencies as a function of the quantum efficiency as obtained when the signal under investigation is a coherent state and for different phase shifts. The two insets show the reconstructed photon distributions for the two phase-modulated versions of the signal corresponding to maximum and minimum visibilities at the output of the Mach-Zehnder interferometer. The vertical black bars denote the mean value of the photon number for the two distributions, $\left\langle a^{\dagger} a\right\rangle$ $=3.5$ and $\left\langle a^{\dagger} a\right\rangle=2.9$. In the lower left plot (left) we report the corresponding reconstructed density matrix in the Fock representation (diagonal and subdiagonal elements). In the lower right plot we report the density matrix for the signal excited in a thermal state.

3 we report the reconstructed distributions at the minimum and maximum of the interference fringes.

\section{ERROR ANALYSIS}

The evaluation of uncertainties on the reconstructed states involves the contributions of experimental fluctuations of on/ off frequencies as well as the statistical fluctuations connected with photon-number reconstruction. It has been argued $[28,29]$ that fluctuations involved in the reconstruction of the photon distribution may generally result in substantial limitations in the information obtainable on the quantum state, e.g., in the case of multipeaked distributions [30]. For our purposes this implies that neither large displacement amplitudes may be employed nor states with large field and/or energy may be reliably reconstructed, although the mean values of the fields measured here are definitely non-negligible.

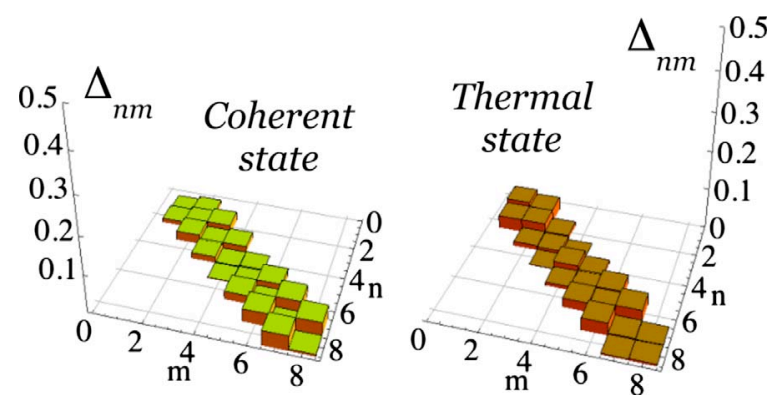

FIG. 4. (Color online) Absolute difference $\Delta_{n m}=\left|\varrho_{n m}^{\text {expt }}-\varrho_{n m}^{\text {theor }}\right|$ between reconstructed and theoretical values of the density matrix elements for the coherent (left) and thermal (right) states used in our experiments.

On the other hand, for the relevant regime of weak field or low energy, observables characterizing the quantum state can be safely evaluated from experimental data, including, e.g., the parity operator used to reconstruct the Wigner function in the phase space. In our experiments, the errors on the reconstructed Wigner function are of the order of the size of the symbols in Fig. 3 whereas the absolute errors $\Delta_{n m}=\mid \varrho_{n m}^{\text {exp }}$ $-\varrho_{n m}^{\text {theor }} \mid$ on the reconstruction of the density matrix in the Fock basis are reported in Fig. 4.

\section{DISCUSSIONS}

We have so far reconstructed the Wigner function and the density matrix for coherent and thermal states. The extension to highly nonclassical states does not require qualitative changes in the setups. The only difference stays in the displacement, which should be obtained with high transmissivity beam splitter in order to avoid mixing of the signal [18].

As our method involves a beam splitter where the signal interferes with a reference state in order to obtain the displacement, we have optimized the effectiveness of mode matching and of the overall scheme by standard visibility test. We note that in this point our technique is similar to QHT, where the signal is amplified by the mixing at a beam splitter with a strong local oscillator. The main difference with standard QHT, however, is the spectral domain of the measurement, which for QHT is confined to the sideband chosen for the measurement, while it is not in our case. The use of pulsed temporal homodyning [31-35] would remove this limitation of QHT. However, this technique is still challenging from the experimental point of view and thus of limited use. The effect of photodetector efficiency should be also taken into account. This is a crucial issue for QHT, which may even prevent effective reconstruction [36]. For the present on/off reconstruction, it does not dramatically affect the accuracy [24]. Notice also that any uncertainty in the nominal efficiency $\eta_{\max }$ of the involved photodetectors does not substantially affect the reconstruction [24].

We stress that our method is especially suited for low excited states, as it does not involve intense fields and delicate balancing to reveal the relevant quantum fluctuations. 


\section{CONCLUSIONS}

In conclusion, we have demonstrated a state reconstruction technique providing Wigner function and density matrix of a field mode starting from on/off photodetection of amplitude- and/or phase-modulated versions of the signal under investigation. Our scheme is little demanding as to the detectors, with the amplitude and phase control required for full state characterization transferred to the optical setup and appears to be reliable and simple especially for states with small number of photons. We foresee a possible widespread use in emerging quantum technologies such as quantum information, metrology, and lithography.

\section{ACKNOWLEDGMENTS}

This work has been partially supported by the CNRCNISM agreement, EU project QuCandela, Compagnia di San Paolo, MIUR-PRIN-2007FYETBY (CCQOTS), and Regione Piemonte (E14).
[1] G. M. D'Ariano, L. Maccone, and M. G. A. Paris, J. Phys. A 34, 93 (2001).

[2] Y. Bogdanov et al., JETP Lett. 82, 164 (2005); C. Marquardt, J. Heersink, R. Dong, M. V. Chekhova, A. B. Klimov, L. L. Sanchez-Soto, U. L. Andersen, and G. Leuchs, Phys. Rev. Lett. 99, 220401 (2007).

[3] D. G. Welsch et al., Prog. Opt. 39, 63 (1999).

[4] Quantum State Estimation, Lecture Notes in Physics Vol. 649, edited by M. G. A. Paris and J. Rehacek (Springer, Berlin, 2004); A. I. Lvovsky and M. G. Raymer, Rev. Mod. Phys. 81, 299 (2009).

[5] D. T. Smithey, M. Beck, M. G. Raymer, and A. Faridani, Phys. Rev. Lett. 70, 1244 (1993).

[6] G. Breitenbach et al., Nature (London) 387, 471 (1997).

[7] M. Vasilyev, S. K. Choi, P. Kumar, and G. Mauro D'Ariano, Phys. Rev. Lett. 84, 2354 (2000)

[8] A. I. Lvovsky, H. Hansen, T. Aichele, O. Benson, J. Mlynek, and S. Schiller, Phys. Rev. Lett. 87, 050402 (2001).

[9] V. Parigi et al., Science 317, 1891 (2008).

[10] V. D’Auria, S. Fornaro, A. Porzio, S. Solimeno, S. Olivares, and M. G. A. Paris, Phys. Rev. Lett. 102, 020502 (2009).

[11] S. Wallentowitz and W. Vogel, Phys. Rev. A 53, 4528 (1996).

[12] K. Banaszek and K. Wodkiewicz, Phys. Rev. Lett. 76, 4344 (1996).

[13] D. Leibfried, D. M. Meekhof, B. E. King, C. Monroe, W. M. Itano, and D. J. Wineland, Phys. Rev. Lett. 77, 4281 (1996).

[14] T. Opatrny and D. G. Welsch, Phys. Rev. A 55, 1462 (1997).

[15] T. Opatrny, D. G. Welsch, and W. Vogel, Phys. Rev. A 56, 1788 (1997).

[16] K. Banaszek, C. Radzewicz, K. Wodkiewicz, and J. S. Krasinski, Phys. Rev. A 60, 674 (1999); Acta Phys. Slov. 49, 643 (1999).

[17] G. Zambra, A. Andreoni, M. Bondani, M. Gramegna, M. Genovese, G. Brida, A. Rossi, and M. G. A. Paris, Phys. Rev. Lett. 95, 063602 (2005); M. Genovese et al., Laser Phys. 16, 385 (2006); G. Brida et al., Open Syst. Inf. Dyn. 13, 333 (2006); M. Bondani, A. Allevi, and A. Andreoni, e-print arXiv:0810.4026.

[18] M. G. A. Paris, Phys. Lett. A 217, 78 (1996).
[19] Z. Hradil, D. Mogilevtsev, and J. Řeháček, Phys. Rev. Lett. 96, 230401 (2006); J. Řeháček, D. Mogilevtsev, and Z. Hradil, New J. Phys. 10, 043022 (2008).

[20] K. E. Cahill and R. J. Glauber, Phys. Rev. 177, 1882 (1969).

[21] G. Zambra, M. Bondani, A. S. Spinelli, and A. Andreoni, Rev. Sci. Instrum. 75, 2762 (2004)

[22] J. Kim, S. Takeuchi, Y. Yamamoto, and H. H. Hogue, Appl. Phys. Lett. 74, 902 (1999); A. Peacock et al., Nature (London) 381, 135 (1996); A. E. Lita et al., Opt. Express 16, 3032 (2008).

[23] G. Di Giuseppe, A. V. Sergienko, B. E. A. Saleh, and M. C. Teich, in Quantum Information and ComputationProceedings of SPIE, edited by E. Donkor, A. R. Pirich, and H. E. Brandt (SPIE,Bellingham WA, 2003), Vol. 5105, p. 39.

[24] A. R. Rossi, S. Olivares, and M. G. A. Paris, Phys. Rev. A 70, 055801 (2004).

[25] G. Brida et al., Opt. Lett. 31, 3508 (2006).

[26] T. Moroder, M. Curty, and N. Lütkenhaus, New J. Phys. 11, 045008 (2009).

[27] K. Laiho, M. Avenhaus, K. N. Cassemiro, and Ch. Silberhorn, New J. Phys. 11, 043012 (2009).

[28] K. Banaszek and K. Wodkiewicz, J. Mod. Opt. 44, 2441 (1997).

[29] K. Banaszek, J. Mod. Opt. 46, 675 (1999).

[30] G. Zambra and M. G. A. Paris, Phys. Rev. A 74, 063830 (2006).

[31] H. Hansen, T. Aichele, C. Hettich, P. Lodahl, A. I. Lvovsky, J. Mlynek, and S. Schiller, Opt. Lett. 26, 1714 (2001).

[32] A. Zavatta, M. Bellini, P. L. Ramazza, F. Marin, and F. T. Arecchi, J. Opt. Soc. Am. B 19, 1189 (2002).

[33] T. Hirano, H. Yamanaka, M. Ashikaga, T. Konishi, and R. Namiki, Phys. Rev. A 68, 042331 (2003).

[34] J. Wenger, R. Tualle-Brouri, and P. Grangier, Opt. Lett. 29, 1267 (2004).

[35] Y. Eto, T. Tajima, Y. Zhang, and T. Hirano, Opt. Lett. 32, 1698 (2007).

[36] G. M. D'Ariano, M. G. A. Paris, and M. F. Sacchi, Adv. Imaging Electron Phys. 128, 205 (2003). 